hep-th/9809065

UCSB NSF-ITP-98-075

McGill/98-12

QMW-PH-98-27

\title{
SUPERSYMMETRY OF ROTATING BRANES
}

\author{
J.P. Gauntlett ${ }^{1}$, R.C. Myers ${ }^{2}$, 1 and P.K. Townsend ${ }^{2}$ 它 \\ ${ }^{1}$ Department of Physics \\ Queen Mary and Westfield College \\ University of London \\ Mile End Road \\ London E1 4NS, UK \\ ${ }^{2}$ Institute for Theoretical Physics \\ University of California at Santa Barbara \\ CA 93106, USA.
}

\begin{abstract}
We present a new $1 / 8$ supersymmetric intersecting M-brane solution of $\mathrm{D}=11$ supergravity with two independent rotation parameters. The metric has a non-singular event horizon and the near-horizon geometry is $a d S_{3} \times S^{3} \times S^{3} \times \mathbb{E}^{2}$ (just as in the non-rotating case). We also present a method of determining the isometry supergroup of supergravity solutions from the Killing spinors and use it to show that for the near horizon solution it is $D(2 \mid 1, \alpha) \times D(2 \mid 1, \alpha)$ where $\alpha$ is the ratio of the two 3 -sphere radii. We also consider various dimensional reductions of our solution, and the corresponding effect of these reductions on the Killing spinors and the isometry supergroups.
\end{abstract}

\footnotetext{
*Permanent address: Department of Physics, McGill University, Montréal, PQ, H3A 2T8, Canada

${ }^{\dagger}$ Permanent address: DAMTP, University of Cambridge, Cambridge CB3 9EW, U.K.
} 


\section{Introduction}

Many of the $a d S_{p+2} \times S^{D-p-2}$ Kaluza-Klein (KK) vacua of $D$-dimensional supergravity theories are known to arise as near-horizon limits of $1 / 2$ supersymmetric $p$-brane solutions [1]. If the $D$-dimensional supergravity is non-maximal then its $p$-brane solutions can usually be viewed as intersections of branes of a maximal supergravity theory, e.g., of Mbranes [2]. An example is the $1 / 2$ supersymmetric self-dual string solution of $(1,1) \mathrm{D}=6$ supergravity, for which the near-horizon geometry is $a d S_{3} \times S^{3}[3]$. This can be interpreted as the $1 / 4$ supersymmetric $(1 \mid M 2, M 5)$ string intersection of an M2-brane with an M5brane, for which the near-horizon limit is a $1 / 2$ supersymmetric $a d S_{3} \times S^{3} \times \mathbb{E}^{5}$ vacuum 41.

The $(1 \mid M 2, M 5)$ intersection is actually a special case of the $1 / 4$ supersymmetric $(1 \mid M 2, M 5, M 5)$ intersection in which the M2-brane intersects two M5-branes. The presence of the M2-brane is associated in this case with a 'generalized harmonic' function of the two sets of M5-brane coordinates [5, 6]. For an appropriate choice of this function there is again a non-singular horizon, near which one finds a $1 / 2$ supersymmetric $a d S_{3} \times S^{3} \times S^{3} \times \mathbb{E}^{2}$ vacuum of $\mathrm{D}=11$ supergravity As argued in [9], and confirmed here, the isometry supergroup of this $D=11$ vacuum solution is

$$
D(2 \mid 1, \alpha)_{L} \times D(2 \mid 1, \alpha)_{R}
$$

where $\alpha$ is the ratio of the radii of the two 3-spheres. Each $D(2 \mid 1, \alpha)$ factor contains an $S l(2 ; \mathbb{R}) \times S U(2) \times S U(2)$ bosonic subgroup, with $\alpha$ being the relative weight of the two $S U(2)$ factors (see e.g. [10). In the limit in which one $S^{3}$ radius goes to infinity we recover the $a d S_{3} \times S^{3} \times \mathbb{E}^{5}$ vacuum.

By adding momentum to the $1 / 4$ supersymmetric $(1 \mid M 2, M 5, M 5)$ configuration (along the string intersection) we arrive at the $1 / 8$ supersymmetric $(1 \mid M W, M 2, M 5, M 5)$ intersection. A curious feature of this case is that the near-horizon limit is again the $1 / 2$ supersymmetric $a d S_{3} \times S^{3} \times S^{3} \times \mathbb{E}^{2}$ vacuum because a wave on $a d S_{3}$ can be removed by a coordinate transformation [11]; this is simply a reflection of the fact that there are no

\footnotetext{
${ }^{1}$ This was originally found as an $a d S_{3} \times S^{3} \times S^{3} \times \mathbb{E}^{1}$ solution of the heterotic string theory [] and was shown in [8] to be the near-horizon limit of a $\mathrm{D}=10 \mathrm{~N}=11 / 4$ supersymmetric $(1 \mid F 1, N S 5, N S 5)$ intersection of a (fundamental) string with two (Neveu-Schwarz) fivebranes.
} 
propagating gravitons in three-dimensions. The main purpose of this paper is to present a new class of $1 / 8$ supersymmetric $(1 \mid M W, M 2, M 5, M 5)$ solutions of $\mathrm{D}=11$ supergravity with two independent rotation parameters. For an appropriate choice of the functions parametrising this class of solution there is a non-singular Killing horizon. The nearhorizon limit is again the $a d S_{3} \times S^{3} \times S^{3} \times \mathbb{E}^{2}$ vacuum. The isometry group is therefore again $D(2 \mid 1, \alpha)_{L} \times D(2 \mid 1, \alpha)_{R}$.

A number of supersymmetric rotating black hole and brane solutions have been found previously. For example, there is a supersymmetric rotating black hole solution of $\mathrm{D}=5$ supergravity $\left[12\right.$, which can interpreted as an $S^{1}$ reduction of a rotating self-dual string solution [13, 14]. It can also be interpreted as the dimensional reduction of a rotating $(0 \mid M 2, M 2, M 2)$ intersection [15]. Many of these rotating brane solutions are related by compactification and/or duality to some special case of our rotating $(1 \mid M W, M 2, M 5, M 5)$ intersection. For example, the rotating (0|M2, M2, M2) intersection is dual to a rotating $(1 \mid M W, M 2, M 5)$ intersection, which can then be dimensionally reduced to yield the rotating self-dual string [15]. But the rotating (1|MW,M2,M5) intersection is just the special case of $(1 \mid M W, M 2, M 5, M 5)$ in which one M5-brane is omitted and its associated rotation parameter set to zero. Thus, the new rotating brane solution presented and analysed here constitutes a generalization of many previous results on rotating branes.

\section{Rotating supersymmetric intersecting M-branes}

Consider two fivebranes and a membrane intersecting according to the pattern

$$
\begin{array}{lllllllllll}
M 5: & 1 & 2 & 3 & 4 & 5 & & & & & \\
M 5: & 1 & & & & & 7 & 8 & 9 & 10 . \\
M 2: & 1 & & & & 6 & & & &
\end{array}
$$

Our new 1/8 supersymmetric M-brane solution, which has momentum flowing along the string intersection and carries angular momentum in the relative transverse directions of the fivebranes $(\{2,3,4,5\}$ and $\{7,8,9,10\})$, can be found within the following general class of solutions of $D=11$ supergravity. The bosonic sector of the $D=11$ supergravity 
Lagrangian is given by 16

$$
\sqrt{-g}\left(R-\frac{1}{12} F^{2}\right)+\frac{2}{(72)^{2}} \epsilon^{M_{1} \cdots M_{4} N_{1} \cdots N_{4} P_{1} P_{2} P_{3}} F_{M_{1} \cdots M_{4}} F_{N_{1} \cdots N_{4}} A_{P_{1} P_{2} P_{3}}
$$

(in these conventions $d \star F+F \wedge F=0$ and the equations of motion are solved by

$$
\begin{aligned}
d s_{11}^{2}= & g_{1}^{1 / 3}\left(g_{2} g_{3}\right)^{2 / 3}\left[\left(g_{1} g_{2} g_{3}\right)^{-1}\left(-2 d u d v+g_{4} d v^{2}+2 A d v\right)\right. \\
& \left.+g_{1}^{-1} d z^{2}+g_{2}^{-1} d x \cdot d x+g_{3}^{-1} d y \cdot d y\right] \\
F^{(4)}= & \frac{c_{1}}{2}\left\{d u d v d g_{1}^{-1}-c_{1} c_{2} \star_{x} d g_{2}-c_{2} \star_{y} d g_{3}+d v \wedge A \wedge d g_{1}^{-1}-g_{1}^{-1} d v \wedge d A\right\} \wedge d z
\end{aligned}
$$

where the functions $g_{1}(x, y), g_{2}(y), g_{3}(x), g_{4}(v, x, y, z)$ and the 1 -form

$$
A=A_{i}(x, y) d x^{i}+A_{\alpha}(x, y) d y^{\alpha}
$$

will be specified below. The constants $c_{1}, c_{2}$ are signs and $\star_{x}$ and $\star_{y}$ are the Hodge duals on the two Euclidean 4-spaces with Cartesian coordinates $x^{i}$ and $y^{\alpha}\left(e . g .,{ }_{x} d g_{2}=\right.$ $\left.(1 / 6) d x^{i} d x^{j} d x^{k} \epsilon^{i j k l} \partial_{l} g_{2}\right)$.

The functions $g_{2}(y)$ and $g_{3}(x)$ are harmonic on their respective $\mathbb{E}^{4}$ spaces and correspond to the two fivebranes. The function $g_{1}(x, y)$ corresponding to the membrane is 'generalized harmonic' [5, 6], i.e., it satisfies

$$
\left[g_{3}^{-1} \nabla_{(x)}^{2}+g_{2}^{-1} \nabla_{(y)}^{2}\right] g_{1}=0
$$

The function $g_{4}(v, x, y, z)$ corresponding to the gravitational wave is also a 'generalized harmonic' function, although in a slightly more general sense than used hitherto. Specifically, it solves the equation

$$
\left[g_{3}^{-1} \nabla_{(x)}^{2}+g_{2}^{-1} \nabla_{(y)}^{2}+g_{1}\left(g_{2} g_{3}\right)^{-1} \partial_{z}^{2}\right] g_{4}=0
$$

The field strength $F=d A$ of the 1 -form $A$ satisfies

$$
\begin{aligned}
& 0=g_{2}^{-1} \partial_{\alpha} F_{\alpha j}+g_{3}^{-1} \partial_{i} F_{i j}+\partial_{i} g_{3}^{-1}\left[F_{i j}+\frac{c_{2}}{2} \epsilon_{i j k l} F_{k l}\right] \\
& 0=g_{3}^{-1} \partial_{i} F_{i \beta}+g_{2}^{-1} \partial_{a} F_{\alpha \beta}+\partial_{\alpha} g_{2}^{-1}\left[F_{\alpha \beta}+\frac{c_{1} c_{2}}{2} \epsilon_{\alpha \beta \gamma \delta} F_{\gamma \delta}\right]
\end{aligned}
$$

One may note the nesting of the lower dimensional objects within the higher dimensional ones: each fivebrane is localized within the worldvolume of the other one (except 
along the string intersection), the membrane is localized within both of the fivebrane worldvolumes, while the wave is localized within the worldvolumes of both fivebranes and the membrane. Note that the dependence of the wave-profile $g_{4}$ on the string direction $v$ is completely arbitrary. This part of the solution is constructed following the standard harmonic function rule [2]. The new aspect of the solution, generalising [17], comes from the introduction of the 1 -form $A$. With an appropriate choice for the solution of eq. (8), one can introduce angular momentum into both of the $\mathbb{E}^{4}$ spaces. In order to maintain supersymmetry, this angular momentum will involve simultaneous rotation in two orthogonal 2-planes within each of these spaces.

Now we discuss the supersymmetries of this $D=11$ supergravity solution. First we write the 11-metric in the form

$$
d s_{11}^{2}=-e^{u} e^{u}+e^{v} e^{v}+e^{z} e^{z}+e^{i} e^{i}+e^{\alpha} e^{\alpha}
$$

with the orthonormal 1-forms

$$
\begin{aligned}
e^{u} & =g_{1}^{-1 / 3}\left(g_{2} g_{3}\right)^{-1 / 6} g_{4}^{-1 / 2}(d u-A) \\
e^{v} & =g_{1}^{-1 / 3}\left(g_{2} g_{3}\right)^{-1 / 6} g_{4}^{1 / 2}\left(d v-g_{4}^{-1}(d u-A)\right) \\
e^{z} & =g_{1}^{-1 / 3}\left(g_{2} g_{3}\right)^{1 / 3} d z \\
e^{i} & =g_{1}^{1 / 6} g_{2}^{-1 / 6} g_{3}^{1 / 3} d x^{i} \\
e^{\alpha} & =g_{1}^{1 / 6} g_{2}^{1 / 3} g_{3}^{-1 / 6} d y^{\alpha}
\end{aligned}
$$

Let $\Gamma^{A}$ with $A=(u, v, z, i, \alpha)$ be the Dirac matrices in this basis. The Killing spinor equations are then

$$
\left\{D+\frac{1}{144}\left[e^{A} \Gamma_{A}^{B C D E}-8 e^{B} \Gamma^{C D E}\right] F_{B C D E}\right\} \epsilon=0
$$

where $D$ is the Lorentz covariant exterior derivative. The solutions are simply written as

$$
\epsilon=g_{1}^{-1 / 6} g_{2}^{-1 / 12} g_{3}^{-1 / 12} g_{4}^{-1 / 4} \epsilon_{0}
$$

where $\epsilon_{0}$ is a constant spinor satisfying the constraints

$$
\Gamma^{u v} \epsilon_{0}=\epsilon_{0}
$$




$$
\begin{aligned}
\Gamma^{z} \epsilon_{0} & =c_{1} \epsilon_{0} \\
\Gamma_{(x)} \epsilon_{0} & =c_{2} \epsilon_{0} \\
\Gamma_{(y)} \epsilon_{0} & =c_{1} c_{2} \epsilon_{0}
\end{aligned}
$$

where

$$
\Gamma_{(x)}=\frac{1}{24} \varepsilon_{i j k l} \Gamma^{i j k l} \quad \Gamma_{(y)}=\frac{1}{24} \varepsilon_{\alpha \beta \gamma \delta} \Gamma^{\alpha \beta \gamma \delta}
$$

The general solution (4) still preserves $1 / 8$ supersymmetry because these four conditions are not all independent. This follows since the 11-dimensional Dirac matrices satisfy $\Gamma^{u v z 12341^{\prime} 2^{\prime} 3^{\prime} 4^{\prime}}=1$.

In order to fully specify a solution of $D=11$ supergravity, we have still to choose the harmonic functions $g_{2}, g_{3}$, solve the 'modified harmonic' equations for $g_{1}$ and $g_{4}$, and solve (8) for $A$. We start by replacing the $x$-coordinates with polar coordinates such that

$$
d s^{2}\left(\mathbb{E}^{4}\right)=d r^{2}+r^{2} d \Omega_{3}^{2}
$$

where $d \Omega_{3}^{2}$ is the metric on the 3 -sphere. A similar primed set of polar coordinates are chosen for the $y$-space. Here, in order facilitate the analysis of the Killing spinors in the next section, we choose coordinates on $S^{3}$ such that

$$
\left.d \Omega_{3}^{2}=\frac{1}{4}\left(d \theta^{2}+d \tilde{\psi}^{2}+d \phi^{2}+2 \cos \theta d \tilde{\psi} d \phi\right)\right)
$$

with

$$
0 \leq \theta<\pi, \quad 0 \leq \phi<2 \pi, \quad 0 \leq \tilde{\psi}<4 \pi
$$

and similarly for the primed coordinates. This metric may be written in the manifestly $S U(2) \times S U(2)$ invariant form

$$
d \Omega_{3}^{2}=\frac{1}{4}\left(\tilde{\sigma}_{1}^{2}+\tilde{\sigma}_{2}^{2}+\tilde{\sigma}_{3}^{2}\right)
$$

where $\tilde{\sigma}_{i}(i=1,2,3)$ are the three left-invariant one-forms on the group $S U(2)$ :

$$
\begin{aligned}
& \tilde{\sigma}_{1}=-\sin \tilde{\psi} d \theta+\cos \tilde{\psi} \sin \theta d \phi \\
& \tilde{\sigma}_{2}=\cos \tilde{\psi} d \theta+\sin \tilde{\psi} \sin \theta d \phi \\
& \tilde{\sigma}_{3}=d \tilde{\psi}+\cos \theta d \phi .
\end{aligned}
$$


We now choose the harmonic functions corresponding to the fivebranes, $g_{2}$ and $g_{3}$, to have single centres:

$$
g_{3}=1+R^{2} / r^{2} \quad g_{2}=1+{R^{\prime}}^{2} / r^{\prime 2}
$$

where constants $R$ and $R^{\prime}$ are related to fivebrane charges. We will consider a solution of the remaining equations such that the singularities at $r=0, r^{\prime}=0$, and $r=r^{\prime}=0$ are not genuine curvature singularities but rather merely coordinate singularities. To this end, we solve (6) by setting

$$
g_{1}=g_{2} g_{3}
$$

In addition we take

$$
g_{4}=1+p g_{2} g_{3}
$$

for some constant $p$. In solving (8), we choose

$$
A=H^{\prime}(y) B(x)+H(x) B^{\prime}(y)
$$

where $H$ and $H^{\prime}$ are harmonic functions,

$$
H=1+\frac{\ell^{2}}{r^{2}} \quad H^{\prime}=1+\frac{\ell^{\prime 2}}{r^{\prime 2}}
$$

and $B$ and $B^{\prime}$ are 1 -forms, on their respective $\mathbb{E}^{4}$ spaces. In order to solve (8), the 2 -form field strengths, $G=d B$ and $G^{\prime}=d B^{\prime}$, must then satisfy

$$
G+\star_{x} G=0 \quad G^{\prime}+\star_{y} G^{\prime}=0
$$

where we have restricted to the case $c_{1}=c_{2}=1$, for convenience. One solution of these anti-self-duality conditions is

$$
B=\frac{J \tilde{\sigma}_{3}}{2 r^{2}} \quad B^{\prime}=\frac{J^{\prime} \tilde{\sigma}_{3}^{\prime}}{2 r^{\prime 2}}
$$

for constants $J$ and $J^{\prime}$, which can be shown (by examination of the asymptotic metric as $r \rightarrow \infty$ and $\left.r^{\prime} \rightarrow \infty\right)$ to correspond to the magnitudes of angular momenta in the $x$ and $y$ 4-spaces. Thus as claimed, we have constructed a rotating $1 / 8$ supersymmetric solution describing a $(1 \mid M W, M 2, M 5, M 5)$ intersection. 


\section{Near-horizon limit}

For the remainder of the paper, we will be considering a near-horizon limit of the preceding solution with both $r / R, r^{\prime} / R^{\prime}<<1$. That is, a limit in which one approaches near both fivebranes simultaneously. Note that the surface $r=r^{\prime}=0$ is at infinite affine distance on spacelike hypersurfaces (with finite $u$ ). One may construct this near-horizon solution with a scaling limit similar to that of [18], and one obtains

$$
\begin{aligned}
d s_{11}^{2}= & \left(R R^{\prime}\right)^{-2}\left[-2\left(r r^{\prime}\right)^{2} d u+\hat{J} \tilde{\sigma}_{3}+\hat{J}^{\prime} \tilde{\sigma}_{3}^{\prime}\right] d v+p d v^{2} \\
& +R^{2} \frac{d r^{2}}{r^{2}}+R^{\prime 2} \frac{d r^{\prime 2}}{r^{\prime 2}}+R^{2} d \Omega_{3}^{2}+R^{\prime 2} d\left(\Omega_{3}^{\prime}\right)^{2}+d z^{2}
\end{aligned}
$$

where $\hat{J}=\ell^{\prime 2} J$ and $\hat{J}^{\prime}=\ell^{2} J^{\prime}$. In terms of the new coordinates

$$
\begin{aligned}
\rho & =r r^{\prime} \\
z^{\prime} & =\frac{R^{2}}{\sqrt{R^{2}+R^{\prime 2}}} \log r-\frac{R^{\prime 2}}{\sqrt{R^{2}+R^{\prime 2}}} \log r^{\prime} \\
w & =\frac{v}{R^{2} R^{\prime 2}} \\
\psi & =\tilde{\psi}+\frac{2 \hat{J}}{R^{2}} w \\
\psi^{\prime} & =\tilde{\psi}^{\prime}+\frac{2 \hat{J}^{\prime}}{R^{\prime 2}} w,
\end{aligned}
$$

the 11-metric becomes

$$
d s_{11}^{2}=-2 \rho^{2} d u d w+\gamma^{2} \frac{d \rho^{2}}{\rho^{2}}+Q^{2} d w^{2}+R^{2} d \Omega_{3}^{2}+R^{\prime 2} d \Omega_{3}^{\prime 2}+d z^{2}+d z^{\prime 2} .
$$

with

$$
Q^{2}=\left(p R^{4} R^{\prime 4}-\frac{\hat{J}^{2}}{R^{2}}-\frac{\hat{J}^{\prime 2}}{R^{\prime 2}}\right)
$$

and

$$
\gamma=\frac{R R^{\prime}}{\sqrt{R^{2}+R^{\prime 2}}}
$$

This metric has the simple form of $a d S_{3} \times S^{3} \times S^{3} \times \mathbb{E}^{2}$ with $\Lambda_{a d S_{3}}=-\gamma^{-2}$. In these coordinates, the near-horizon four-form field strength is given by

$$
F=d z \wedge\left(\frac{\operatorname{Vol}\left(S_{R}^{3}\right)}{R}+\frac{\operatorname{Vol}\left(S_{R^{\prime}}^{3}\right)}{R^{\prime}}-\frac{\operatorname{Vol}\left(\operatorname{ad} S_{3}\right)}{\gamma}\right)
$$


where $\operatorname{Vol}(X)$ denotes the volume form on the corresponding three-dimensional space $X$.

Note that up to this point we have not considered any of the coordinates to be periodically identified, other than the 3-sphere identifications (17). Thus there are no global subtleties in implementing the above coordinate transformation (28). In particular, one finds that the remnant of the angular momentum in this near-horizon limit is a coordinate artifact because the transition from $\tilde{\psi}, \tilde{\psi}^{\prime}$ to $\psi, \psi^{\prime}$ removes all mixing of $v$ with the angular coordinates. This generalizes a similar observation made recently in the context of the near-horizon limit of certain spinning five-dimensional black holes [14]. There is still a remnant of the gravitational wave in the near-horizon metric in the form of the $d w^{2}$ term. This can also be removed by a coordinate transformation [11] but we choose not to do this here because compactifications will typically require periodic identification of $w$, in which case the coordinate transformation that removes the $d w^{2}$ term could only be implemented locally. In general, the angular momenta and gravitational wave of the full solution will leave their imprint in the near-horizon solution in the form of the global identifications which complete the specification of the compactified geometry.

\section{Near-horizon Killing spinors}

Now we wish to determine the supersymmetry enhancement which arises in the nearhorizon limit above by explicitly solving for the Killing spinors. Recall that the Killing spinors satisfy

$$
\left\{D+\frac{1}{144}\left[e^{A} \Gamma_{A}^{B C D E}-8 e^{B} \Gamma^{C D E}\right] F_{B C D E}\right\} \epsilon=0
$$

where the covariant exterior derivative is given by

$$
D=d+\frac{1}{4} e^{A} \omega_{A B C} \Gamma^{B C} \equiv d+e^{A} M_{A}
$$

A suitable choice of frame 1-forms for the near-horizon 11-metric (29) is

$$
\begin{aligned}
e^{u} & =\frac{\rho^{2}}{Q} d u \\
e^{w} & =Q d w-\frac{\rho^{2}}{Q} d u \\
e^{\rho} & =\gamma \frac{d \rho}{\rho}
\end{aligned}
$$




$$
\begin{array}{rlrl}
e^{i} & =\frac{R}{2} \sigma_{i} & & (i=1,2,3) \\
e^{\alpha} & =\frac{R^{\prime}}{2} \sigma_{\alpha}^{\prime} & & \left(\alpha=1^{\prime}, 2^{\prime}, 3^{\prime}\right) \\
e^{z} & =d z & e^{z^{\prime}}=d z^{\prime}
\end{array}
$$

where the forms $\sigma_{i}$ are defined as in (19) with $\theta, \phi, \psi$, and similarly for the primed angles. The matrices $M_{A}$ appearing in the covariant derivative (34) are

$$
\begin{array}{ll}
M_{u} & =-\frac{1}{2 \gamma} \Gamma^{w \rho}-\frac{1}{\gamma} \Gamma^{u \rho} \\
M_{w} & =-\frac{1}{2 \gamma} \Gamma^{u \rho} \\
M_{\rho} & =\frac{1}{2 \gamma} \Gamma^{w u} \\
M_{1} & =\frac{1}{2 R} \Gamma^{23} \quad \text { (and cyclic) } \\
M_{1^{\prime}} & =\frac{1}{2 R^{\prime}} \Gamma^{2^{\prime} 3^{\prime}} \quad \text { (and cyclic) }
\end{array}
$$

while $M_{z}=0=M_{z^{\prime}}$.

We now define matrices $N_{A}$ by

$$
e^{A} N_{A} \equiv \frac{1}{144}\left(e^{A} \Gamma_{A}^{B C D E}-8 e^{B} \Gamma^{C D E}\right) F_{B C D E}
$$

so that the Killing spinor equations (33) become $\left(\partial_{\mu}+e_{\mu}^{A}\left(M_{A}+N_{A}\right)\right) \epsilon=0$. In particular, using (32) one has

$$
N_{z}=-\frac{1}{3}\left[\frac{1}{R} \Gamma^{123}+\frac{1}{R^{\prime}} \Gamma^{1^{\prime} 2^{\prime} 3^{\prime}}-\frac{1}{\gamma} \Gamma^{u w \rho}\right]
$$

Therefore the Killing spinors $\epsilon$ will be independent of $z$ provided that $N_{z} \epsilon=0$, which is equivalent to

$$
\Gamma \epsilon=\epsilon
$$

where

$$
\Gamma=\frac{\gamma}{R} \Gamma^{u w \rho 123}+\frac{\gamma}{R^{\prime}} \Gamma^{u w \rho 1^{\prime} 2^{\prime} 3^{\prime}}
$$

Note that $\Gamma^{2}=1$. One also finds that $N_{z^{\prime}}=(1 / 2) \Gamma^{z z^{\prime}} N_{z}$. Therefore the constraint (39) also guarantees that the Killing spinors are independent of $z^{\prime}$. The remaining $N_{A}$ are

$$
N_{u}=\left(\frac{1}{6 \gamma} \Gamma^{\rho w} \Gamma+\frac{1}{3 \gamma} \Gamma^{\rho w}\right) \Gamma^{z}
$$




$$
\begin{aligned}
& N_{w}=\left(\frac{1}{6 \gamma} \Gamma^{u \rho} \Gamma+\frac{1}{3 \gamma} \Gamma^{u \rho}\right) \Gamma^{z} \\
& N_{\rho}=\left(\frac{1}{6 \gamma} \Gamma^{w u} \Gamma+\frac{1}{3 \gamma} \Gamma^{w u}\right) \Gamma^{z} \\
& N_{1}=\left(\frac{1}{6 \gamma} \Gamma^{1 w \rho u}[1-\Gamma]+\frac{1}{2 R} \Gamma^{23}\right) \Gamma^{z} \quad \text { (and cyclic) } \\
& N_{1^{\prime}}=\left(\frac{1}{6 \gamma} \Gamma^{1^{\prime} w \rho u}[1-\Gamma]+\frac{1}{2 R^{\prime}} \Gamma^{2^{\prime} 3^{\prime}}\right) \Gamma^{z} \quad \text { (and cyclic) }
\end{aligned}
$$

On the $\Gamma=1$ eigenspace the remaining Killing spinor equations now become

$$
\begin{aligned}
0 & =\left(\partial_{u}-\frac{\rho^{2}}{Q \gamma}\left(\Gamma^{w \rho}-\Gamma^{\rho u}\right) \Gamma_{+}\right) \epsilon \\
0 & =\left(\partial_{w}-\frac{Q}{\gamma} \Gamma^{u \rho} \Gamma_{-}\right) \epsilon \\
0 & =\left(\partial_{\rho}+\rho^{-1} \Gamma^{w u} \Gamma_{+}\right) \epsilon \\
0 & =\left(\partial_{\theta}+\frac{1}{2}\left(\sin \psi \Gamma^{32}+\cos \psi \Gamma^{31}\right) \Gamma_{+}\right) \epsilon \\
0 & =\left(\partial_{\phi}+\frac{1}{2}\left(\cos \psi \sin \theta \Gamma^{23}+\sin \psi \sin \theta \Gamma^{31}+\cos \theta \Gamma^{12}\right) \Gamma_{+}\right) \epsilon \\
0 & =\left(\partial_{\psi}+\frac{1}{2} \Gamma^{12} \Gamma_{+}\right) \epsilon
\end{aligned}
$$

and similarly for the equations in the primed angles, where we have defined the projection operators

$$
\Gamma_{ \pm}=\frac{1}{2}\left(1 \pm \Gamma^{z}\right)
$$

The solution can be expressed in terms of a constant spinor $\chi$ subject to the single constraint (39)

$$
\Gamma \chi=\chi
$$

As $\Gamma$ commutes with both $\Gamma^{z}$ and $\Gamma^{w u}$ this condition is compatible with the decomposition

$$
\chi=\left(\chi_{+}^{+}+\chi_{+}^{-}\right)+\left(\chi_{-}^{+}+\chi_{-}^{-}\right)
$$

where

$$
\Gamma^{z} \chi_{ \pm}= \pm \chi_{ \pm} \quad \Gamma^{w u} \chi^{ \pm}= \pm \chi^{ \pm}
$$

Since $\Gamma_{+}$annihilates $\chi_{-}$, the corresponding Killing spinors are independent of all coordinates except $w$. The $w$ equation is solved by

$$
\epsilon_{-}=e^{\frac{Q w}{\gamma} \Gamma^{u \rho}}\left(\chi_{-}^{+}+\chi_{-}^{-}\right)
$$


Similarly $\Gamma_{-}$annihilates $\chi_{+}$, so the corresponding Killing spinors are independent of $w$. The remaining equations (42) are solved by

$$
\epsilon_{+}=\left(\frac{1}{\rho}-\frac{2 u \rho}{Q \gamma} \Gamma^{\rho u}\right) \Omega \Omega^{\prime} \chi_{+}^{+}+\rho \Omega \Omega^{\prime} \chi_{+}^{-}
$$

where

$$
\Omega(\theta, \phi, \psi)=e^{\frac{1}{2} \Gamma^{21} \psi} e^{\frac{1}{2} \Gamma^{13} \theta} e^{\frac{1}{2} \Gamma^{21} \phi}
$$

and similarly for $\Omega^{\prime}\left(\theta^{\prime}, \phi^{\prime}, \psi^{\prime}\right)$.

Hence given the single constraint (39), we conclude that the near-horizon solution, (29) and (32), preserves one-half the supersymmetries. This represents a four-fold increase over that for the full solution presented in the first section. Of course this counting assumes that only the angular coordinates have periodic identifications. With a reduction on $w$, (naively at least) the $w$ dependence of $\epsilon_{-}$rules these out as admissible supersymmetries, and the near-horizon solution would only preserve one-quarter the supersymmetries, as expected. We will discuss this further in section 7 .

Recall that the determination of Killing spinors in section 2 was done for the general solution (4) without a particular choice of metric functions. For the purpose of comparing with the present results it is convenient to repeat this analysis in polar coordinates (15)). With the assumption that the only $x^{i}$ and $y^{\alpha}$ dependence is on the respective radial coordinates, $r$ and $r^{\prime}$, i.e., $g_{1}=g_{1}\left(r, r^{\prime}\right), g_{2}=g_{2}\left(r^{\prime}\right), g_{3}=g_{3}(r), g_{4}=g_{4}\left(r, r^{\prime}, z\right)$, one finds (12) is replaced by

$$
\epsilon=g_{1}^{-1 / 6} g_{2}^{-1 / 12} g_{3}^{-1 / 12} g_{4}^{-1 / 4} \Omega \Omega^{\prime} \epsilon_{0} .
$$

Hence if we look at the near-horizon limit of these spinors, we find

$$
\epsilon=r r^{\prime} \Omega \Omega^{\prime} \epsilon_{0}=\rho \Omega \Omega^{\prime} \epsilon_{0} .
$$

So as expected, we see that these Killing spinors match up to the $\epsilon_{+}^{-}$spinors in (48). That is, only $\epsilon_{+}^{-}$supersymmetries may be extended into the asymptotic regions of the solution, e.g., large $\rho$. 


\section{$5 \quad$ Killing spinors and the isometry superalgebra}

Any supergravity solution can be presented as a configuration of tensors on superspace. The local isometry supergroup of the solution is, by definition, the supergroup generated by the Killing vector superfields, i.e. those vector superfields which leave the superspace configuration invariant (see [19 for details). It might seem that a determination of the isometry superalgebra of a given supergravity solution would require that one first find its superspace presentation, which is a very laborious task. Fortunately, a shortcut is possible, at least for solutions that are purely bosonic. The method, which we explain below, relies on the fact that any pair of Killing spinors determines a Killing vector field. We shall now explain this point.

We begin by noting that given two $\mathrm{D}=11$ Killing spinors, $\zeta$ and $\zeta^{\prime}$, then the $\mathrm{D}=11$ vector field

$$
v=\bar{\zeta} \Gamma^{M} \zeta^{\prime} \partial_{M}
$$

is Killing. The proof is as follows: We first observe that

$$
\overline{\Gamma_{(n)} \zeta}=(-1)^{\frac{n(n+1)}{2}} \bar{\zeta} \Gamma_{(n)}
$$

where $\Gamma_{(n)}$ is the antisymmetrized product of $n$ of the Dirac matrices $\Gamma^{A}$. It follows, for any spinor $\zeta$, that

$$
\overline{D_{M} \zeta}=\partial_{M} \bar{\zeta}-\frac{1}{4} \bar{\zeta} \Gamma^{A B} \omega_{M A B}=D_{M} \bar{\zeta}
$$

and that a Killing spinor $\zeta$ satisfies

$$
\overline{D_{M} \zeta}=\frac{1}{144} \bar{\zeta}\left[\Gamma_{M}{ }^{N P Q R}+8 \delta_{M}^{N} \Gamma^{P Q R}\right] F_{N P Q R}
$$

It further follows from (54) that

$$
D_{M} v_{S}=\overline{D_{M} \zeta} \Gamma_{S} \zeta^{\prime}+\bar{\zeta} \Gamma_{S} D_{M} \zeta^{\prime}
$$

Using the Killing spinor condition in both terms on the right hand side we deduce that

$$
D_{M} v_{S}=\frac{1}{144} \bar{\zeta}\left[2 \Gamma_{M}{ }^{N P Q R}{ }_{S}+48 \delta_{M}^{N} \delta_{S}{ }^{P} \Gamma^{Q R}\right] \zeta^{\prime} F_{N P Q R}
$$

and hence that

$$
D_{(M} v_{S)}=0
$$


as required for a Killing vector field.

We now turn to the explanation how the above observations can be used to determine the isometry superalgebra of a given supergravity solution (and hence its local isometry supergroup) from a knowledge of its Killing spinors. An expansion of the $\mathrm{D}=11$ supergravity action about the solution of interest yields currents $\left(T^{m n}, j_{\alpha}^{m}, K^{m n p}\right)$ with background covariant conservation conditions determined by the gauge transformations of the fluctuation fields. These currents must belong to a supermultiplet with respect to the supersymmetries of the background associated with Killing spinors. This statement can be formalized in terms of the once-integrated current algebra anticommutator

$$
\left\{Q_{F}(\zeta), j^{m}\right\}=\frac{1}{2} T^{m n} \Gamma_{n} \zeta+\frac{1}{2} K^{m n p} \Gamma_{n p} \zeta
$$

where

$$
Q_{F}(\zeta)=\int_{\Sigma} d S_{m} \bar{\zeta} j^{m}
$$

is the (Fermionic) charge of the fluctuation fields associated with the background Killing spinor $\zeta$. For fluctuation fields that fall off sufficiently rapidly towards the boundary at infinity on the spacelike hypersurface $\Sigma$, these charges will be time-independent by virtue of the conservation condition on $j^{m}$ and the Killing spinor condition obeyed by $\zeta$.

Integrating the relation (59) and discarding the integral over $K^{m n p}$ on the grounds that it could be non-zero only in the presence of a membrane source, we find that

$$
\left\{Q_{F}(\zeta), Q_{F}\left(\zeta^{\prime}\right)\right\}=Q_{B}\left(v=\zeta^{\prime} \Gamma \zeta\right)
$$

where

$$
Q_{B}(v)=\int_{\Sigma} d S_{m} v^{n} T_{n}^{m}
$$

Since $v$ is Killing this (Bosonic) charge is also time-independent. What this shows is that the determination of the linear combination of Killing vector fields associated with any pair of Killing spinors is equivalent to the determination of the linear combination of bosonic charges in the isometry superalgebra that appear in the anticommutator of any pair of fermionic charges in this algebra.

We shall now use this method to determine the isometry superalgebra of the nearhorizon limit of our new rotating brane solution and of some of its special cases. Of 
course, there may be additional bosonic isometries that are not found in the above way. In this case the full isometry superalgebra will be the direct product of the superalgebra as determined by the above method with a purely bosonic algebra.

\section{The isometry supergroup of $A d S_{3} \times S^{3} \times S^{3}$}

Using the arguments of the last section we conclude that to determine the isometry supergroup of the near horizon geometry of our rotating brane solution, which is simply $A d S_{3} \times S^{3} \times S^{3}$, we need to construct the Killing vectors associated to the Killing spinors (47) and (48). We will show that the answer is given by $D(2 \mid 1, \alpha) \times D(2 \mid 1, \alpha)$

To begin, we first observe that for any two Killing spinors $\epsilon, \epsilon^{\prime}$ we have

$$
\bar{\epsilon} \Gamma^{z} \epsilon^{\prime}=\bar{\epsilon} \Gamma^{z^{\prime}} \epsilon^{\prime}=0
$$

since they lie on the $\Gamma=1$ eigenspace. In addition we have

$$
\bar{\epsilon}_{+} \Gamma^{A} \epsilon_{-}=0
$$

for all $A$ (using, for example, $\left\{\Gamma^{A}, \Gamma^{z}\right\}=0$ for $A \neq z$ ). It follows that the only Killing vectors obtainable from Killing spinors are

$$
v_{++}=\left(v_{++}^{++}, v_{++}^{+-}, v_{++}^{--}\right) \quad \text { and } \quad v_{--}=\left(v_{--}^{++}, v_{--}^{+-}, v_{--}^{--}\right)
$$

where, e.g., $v_{--}^{+-}=\bar{\epsilon}_{-}^{+} \Gamma^{A} \epsilon_{-}^{-} \tilde{e}_{A}$. 2 Here, we are using the dual basis vectors $\tilde{e}_{A}$

$$
\begin{aligned}
\tilde{e}_{u} & =Q \rho^{-2} \partial_{u}+Q^{-1} \partial_{w} \\
\tilde{e}_{w} & =Q^{-1} \partial_{w} \\
\tilde{e}_{\rho} & =\frac{\rho}{\gamma} \partial_{\rho} \\
\tilde{e}_{i} & =\frac{2}{R} \xi_{i}^{R} \\
\tilde{e}_{\alpha} & =\frac{2}{R^{\prime}} \xi_{\alpha}^{R} \quad \tilde{e}_{z^{\prime}}=\partial_{z^{\prime}}
\end{aligned}
$$

\footnotetext{
${ }^{2}$ Note that our notation here means, for example, $\epsilon_{+}^{-}$is the Killing spinor depending on $\chi_{+}^{-}$. However, unlike $\chi_{+}^{-}, \epsilon_{+}^{-}$does not have a simple projection under $\Gamma^{w u}$, i.e., $\Gamma^{w u} \epsilon_{-}^{+} \neq+\epsilon_{-}^{+}$.
} 
where $\xi^{R}$ are left-invariant vector fields on each $S^{3}$ dual (that generate right actions) which are dual to the left-invariant one-forms $\sigma$ and whose explicit form is

$$
\begin{aligned}
& \xi_{1}^{R}=-\sin \psi \partial_{\theta}+\cos \psi \operatorname{cosec} \theta \partial_{\phi}-\cot \theta \cos \psi \partial_{\psi} \\
& \xi_{2}^{R}=\cos \psi \partial_{\theta}+\sin \psi \operatorname{cosec} \theta \partial_{\phi}-\cot \theta \sin \psi \partial_{\psi} \\
& \xi_{3}^{R}=\partial_{\psi} .
\end{aligned}
$$

Later the right-invariant vector fields $\xi^{L}$ (that generate left actions) will also appear. They are given by

$$
\begin{aligned}
\xi_{1}^{L} & =\sin \phi \partial_{\theta}+\cot \theta \cos \phi \partial_{\phi}-\cos \phi \operatorname{cosec} \theta \partial_{\psi} \\
\xi_{2}^{L} & =\cos \phi \partial_{\theta}-\cot \theta \sin \phi \partial_{\phi}+\sin \phi \operatorname{cosec} \theta \partial_{\psi} \\
\xi_{3}^{L} & =\partial_{\phi} .
\end{aligned}
$$

The commutation relations are given by

$$
\left[\xi_{i}^{R}, \xi_{j}^{R}\right]=-\epsilon_{i j k} \xi_{k}^{R} \quad\left[\xi_{i}^{L}, \xi_{j}^{L}\right]=\epsilon_{i j k} \xi_{k}^{L} \quad\left[\xi_{i}^{R}, \xi_{j}^{L}\right]=0
$$

There are a number of useful identities in the computation of the Killing vectors. First, we observe the fact that

$$
\bar{\chi}^{ \pm} \Gamma^{\rho} \chi^{ \pm}=\bar{\chi}^{ \pm} \Gamma^{i} \chi^{ \pm}=\bar{\chi}^{ \pm} \Gamma^{\alpha} \chi^{ \pm}=0
$$

because $\Gamma^{\rho}, \Gamma^{i}$ and $\Gamma^{\alpha}$ all commute with $\Gamma^{w u}$. Also

$$
\bar{\chi}^{ \pm} \Gamma^{u} \chi^{\mp}=\bar{\chi}^{ \pm} \Gamma^{w} \chi^{\mp}=0
$$

because $\Gamma^{u}$ and $\Gamma^{w}$ anticommute with $\Gamma^{w u}$. Similarly

$$
\bar{\chi}^{ \pm} \Gamma^{w \rho i} \chi^{\mp}=0 \quad \bar{\chi}^{ \pm} \Gamma^{w \rho \alpha} \chi^{\mp}=0
$$

Finally note from the definition of the superscript indices on the constant spinors that $\Gamma^{w} \chi^{ \pm}= \pm \Gamma^{u} \chi^{ \pm}$

Now a calculation yields

$$
\begin{aligned}
v_{--}^{++} & =\frac{2}{\gamma}\left(\bar{\chi}_{-}^{+} \Gamma^{u} \chi_{-}^{+}\right) \ell_{+}^{R} \\
v_{--}^{+-} & =\frac{2}{\gamma}\left(\bar{\chi}_{-}^{+} \Gamma^{\rho} \chi_{-}^{-}\right) \ell_{0}^{R}+\frac{2}{R}\left(\bar{\chi}_{-}^{+} \Gamma^{i} \chi_{-}^{-}\right) \xi_{i}^{R}+\frac{2}{R^{\prime}}\left(\bar{\chi}_{-}^{+} \Gamma^{\alpha} \chi_{-}^{-}\right) \xi_{\alpha}^{R} \\
v_{--}^{--} & =\frac{2}{\gamma}\left(\bar{\chi}_{-}^{-} \Gamma^{u} \chi_{-}^{-}\right) \ell_{-}^{R}
\end{aligned}
$$


where

$$
\begin{aligned}
\ell_{ \pm}^{R} & =\frac{\gamma Q}{2 \rho^{2}} \cosh \frac{2 Q w}{\gamma} \partial_{u}+\frac{\gamma}{2 Q}\left(\cosh \frac{2 Q w}{\gamma} \pm 1\right) \partial_{w}-\frac{\rho}{2} \sinh \frac{2 Q w}{\gamma} \partial_{\rho} \\
\ell_{0}^{R} & \left.=-\frac{\gamma Q}{2 \rho^{2}} \sinh \frac{2 Q w}{\gamma} \partial_{u}-\frac{\gamma}{2 Q} \sinh \frac{2 Q w}{\gamma} \partial_{w}+\frac{\rho}{2} \cosh \frac{2 Q w}{\gamma} \partial_{\rho}\right)
\end{aligned}
$$

These obey the $S O(2,1)$ commutation relations

$$
\left[\ell_{+}^{R}, \ell_{-}^{R}\right]=-2 \ell_{0}^{R} \quad\left[\ell_{0}^{R}, \ell_{ \pm}^{R}\right]= \pm \ell_{ \pm}^{R}
$$

The $v_{++}$Killing vectors are

$$
\begin{aligned}
v_{++}^{++} & =\frac{2}{\gamma}\left(\bar{\chi}_{+}^{+} \Gamma^{u} \chi_{+}^{+}\right) \ell_{+}^{L} \\
v_{++}^{+-} & =\frac{2}{\gamma}\left(\bar{\chi}_{+}^{+} \Gamma^{\rho} \chi_{+}^{-}\right) \ell_{0}^{L}+\frac{2}{R}\left(\bar{\chi}_{+}^{+} \Gamma^{i} \chi_{+}^{-}\right) \xi_{i}^{L}+\frac{2}{R^{\prime}}\left(\bar{\chi}_{+}^{+} \Gamma^{\alpha} \chi_{+}^{-}\right) \xi_{\alpha}^{L} \\
v_{++}^{--} & =\frac{2}{\gamma}\left(\bar{\chi}_{+}^{-} \Gamma^{u} \chi_{+}^{-}\right) \ell_{-}^{L}
\end{aligned}
$$

where

$$
\begin{aligned}
\ell_{+}^{L} & =\left(\frac{\gamma Q}{2 \rho^{4}}+\frac{2 u^{2}}{Q \gamma}\right) \partial_{u}+\frac{\gamma}{Q \rho^{2}} \partial_{w}-\frac{2 u \rho}{Q \gamma} \partial_{\rho} \\
\ell_{-}^{L} & =\frac{\gamma Q}{2} \partial_{u} \\
\ell_{0}^{L} & =\frac{\rho}{2} \partial_{\rho}-u \partial_{u}
\end{aligned}
$$

which satisfy the standard $S O(2,1)$ commutation relations

$$
\left[\ell_{+}^{L}, \ell_{-}^{L}\right]=2 \ell_{0}^{L} \quad\left[\ell_{0}^{L}, \ell_{ \pm}^{L}\right]=\mp \ell_{ \pm}^{L}
$$

and in addition commute with the $R$ generators. In deriving $(\sqrt{76})$ we have used the fact that

$$
\Omega^{-1} \Gamma^{i} \Omega \equiv \Gamma^{j} R_{j}^{i}(\Omega)
$$

with

$$
R_{j}^{i}(\Omega) \xi_{i}^{R}=\xi_{j}^{L}
$$

From (73) and (76) we conclude that the near-horizon isometry supergroup is given by

$$
D(2 \mid 1, \alpha)_{L} \times D(2 \mid 1, \alpha)_{R}
$$


where

$$
\alpha=\frac{R^{\prime}}{R}
$$

which is the ratio of the radii of the two 3 -spheres.

\section{$7 \quad$ Killing spinors and reduction}

One might consider constructing solutions of lower-dimensional supergravity from $\mathrm{D}=11$ supergravity solutions when the latter have Killing symmetries. Here, we wish to consider the effect of such dimensional reduction on the Killing spinors. Naively, one expects that the only Killing spinors to survive will be those which are independent of the internal coordinates on which one is reducing. More precisely, when reducing on a Killing vector $k$, we must require that the Killing spinors have a vanishing Lie derivative under $k$. The Lie derivative of a spinor $\epsilon$ with respect to an arbitrary vector field is ill-defined, but with respect to a Killing vector field it is given by (see, e.g., [5])

$$
\mathcal{L}_{k} \epsilon=i_{k} D \epsilon+\frac{1}{8} \Gamma^{m n}(d k)_{m n} \epsilon
$$

where $D$ is the covariant derivative defined in (34), and $(d k)$ is the exterior derivative of the 1-form $k_{m} d x^{m}$ dual to $k^{m} \partial_{m}$. Killing spinors satisfy the supersymmetry Killing equations: $\left(D_{m}+N_{m}\right) \epsilon=0$. Thus, a vanishing Lie derivative of $\epsilon$ implies

$$
\begin{aligned}
0 & =k^{m} D_{m} \epsilon+\frac{1}{8} \Gamma^{m n}(d k)_{m n} \epsilon \\
& =\left(\frac{1}{8} \Gamma^{m n}(d k)_{m n}-k^{m} N_{m}\right) \epsilon \equiv P \epsilon .
\end{aligned}
$$

In other words, the vanishing Lie derivative condition reduces to a simple algebraic $\Gamma$ matrix constraint $(P \epsilon=0)$ on the Killing spinors. Dimensional reduction will therefore reduce the number of Killing spinors to those satisfying this constraint. The details will, of course, depend on the solution and the particular choice of $k$, so we shall illustrate the procedure with a number of simple examples. 


\subsection{Zero Angular Momentum}

The non-rotating near-horizon metric (29) may be written as

$$
\begin{aligned}
d s_{11}^{2}=-( & \left.\frac{\rho^{2}}{Q} d u\right)^{2}+\left(Q d w-\frac{\rho^{2}}{Q} d u\right)^{2}+\gamma^{2}\left(\frac{d \rho}{\rho}\right)^{2} \\
& +\frac{R^{2}}{4}\left(\sigma_{1}^{2}+\sigma_{2}^{2}+\sigma_{3}^{2}\right)+\frac{R^{\prime 2}}{4}\left(\sigma_{1}^{\prime 2}+\sigma_{2}^{\prime 2}+\sigma_{3}^{\prime 2}\right)+d z^{2}+d z^{\prime 2}
\end{aligned}
$$

There are a number of simple Killing vectors upon which we will consider reducing the solution: $\partial_{z}, \partial_{z^{\prime}}, \partial_{w}, \partial_{\psi}$ and $\partial_{\psi^{\prime}}$. Note that demanding that (any of the first three of) these Killing vectors have closed orbits, will imply global identifications on the $a d S_{3} \times$ $S^{3} \times S^{3} \times \mathbb{E}^{2}$ geometry.

The reduction on $\partial_{z}$ or $\partial_{z^{\prime}}$ is trivial. In this case, $d k=0$ and so the constraint (84) reduces to $P \epsilon=-N_{z, z^{\prime}} \epsilon=0$. However, this is equivalent to the constraint (39) already imposed on all of the Killing spinors. Of course, (39) was derived from requiring the Killing spinors be independent of $z$ and $z^{\prime}$. Hence it is no surprise that all of the Killing spinors survive unchanged when the theory is reduced on these two directions, i.e., there is no reduction in the number of supersymmetries.

Next consider a reduction on $\partial_{w}$. First let us note that the metric has precisely the one that one would adopt for a standard Kaluza-Klein compactification for a reduction on $w$. The reduced metric would correspond to (85) without the $\left(e^{w}\right)^{2}$ term, i.e.,

$$
d s^{2}=-\left(\frac{\rho^{2}}{Q} d u\right)^{2}+\gamma^{2}\left(\frac{d \rho}{\rho}\right)^{2}+\ldots
$$

and the off-diagonal component $g_{w u}$ would become a gauge field $-\rho^{2} / Q d u$ in the lowerdimensional theory. As is apparent in (86), adS $S_{3}$ is replaced by $a d S_{2}$ in the reduced geometry. If the frame (35) (without $e^{w}$ ) is chosen to describe the reduced geometry, the form of the Killing spinors will be unchanged up to the additional constraint (84). In this case, we have $k=Q \tilde{e}_{w}$, and as a 1 -form, $k=Q e^{w}$. Hence one finds $d k=\frac{2 Q}{\gamma} e^{u} e^{\rho}$, and

from (84) $P=\frac{Q}{\gamma} \Gamma^{u \rho} \Gamma_{-}$. Hence the Killing spinors surviving the reduction must satisfy $\Gamma_{-} \epsilon=0$ which picks out the $\epsilon_{+}$spinors in (48). This result then agrees with the naive expectation that one should chose the spinors independent of $w$.

Reducing on $\partial_{\psi}$ also produces an interesting lower-dimensional solution. (Of course, a reduction on $\partial_{\psi^{\prime}}$ completely parallels the following analysis.) Recalling the definition (19) 
for the $\sigma_{i}$ 's, we note that the metric (85) is again adapted for a Kaluza-Klein reduction on $\psi$. In this case, the reduced metric becomes

$$
\begin{aligned}
d s^{2} & =\frac{R^{2}}{4}\left(\sigma_{1}^{2}+\sigma_{2}^{2}\right)+\ldots \\
& =\frac{R^{2}}{4}\left(d \theta^{2}+\sin ^{2} \theta d \phi^{2}\right)+\ldots
\end{aligned}
$$

and so one of the $S^{3}$ factors is replaced by $S^{2}$ in the reduced geometry. The latter also carries a monopole gauge field arising from $g_{\psi \phi}$. Here, we have $k=(R / 2) \tilde{e}_{3}$, and as a 1-form, $k=(R / 2) e^{3}$. Then (84) yields $P=\frac{1}{2} \Gamma^{12} \Gamma_{-}$, and so the Killing spinors surviving this reduction again satisfy $\Gamma_{-} \epsilon=0$. Thus the $\epsilon_{+}$spinors correspond to supersymmetries in the reduced geometry.

At first sight, this is a surprise since it is the $\epsilon_{-}$spinors (47) which are independent of $\psi$. The resolution of this puzzle comes from realizing that $e^{1}$ and $e^{2}$ can not be used as orthonormal 1-forms in the reduced theory, as they are $\psi$ dependent - see (19). Rather they should be replaced by the 1 -forms, e.g.,

$$
\hat{e}^{1}=\frac{R}{2} \sin \theta d \phi \quad \hat{e}^{2}=\frac{R}{2} d \theta .
$$

In this case, the two sets of 1 -forms are related by a simple rotation acting in the 1-2 subspace

$$
\hat{e}^{a}=L_{b}^{a} e^{b} \quad \text { with } \quad L=\left(\begin{array}{cc}
\cos \psi & \sin \psi \\
-\sin \psi & \cos \psi
\end{array}\right)=\exp \left[\left(\begin{array}{cc}
0 & 1 \\
-1 & 0
\end{array}\right) \psi\right]
$$

If Lorentz vectors are rotated by $L_{b}^{a}=\exp \left(\omega^{a}{ }_{b}\right)$, then the corresponding transformation of spinors is

$$
\widetilde{L}=\exp \left(\frac{1}{4} \omega_{a b} \Gamma^{a b}\right)
$$

Specifically for (89), we have

$$
\widetilde{L}_{\psi}=\exp \left(-\frac{1}{2} \Gamma^{21} \psi\right)
$$

Hence reducing on $\partial_{\psi}$ requires modifying the frame, and in doing so the precise form of the Killing spinors changes by

$$
\hat{\epsilon}=\widetilde{L}_{\psi} \epsilon=e^{-\frac{1}{2} \Gamma^{21} \psi} \epsilon
$$


However, this transformation precisely cancels the $\psi$ dependence of the $\epsilon_{+}$spinors, and thus the $\hat{\epsilon}_{+}$appear as Killing spinors in the reduced theory. Here, we should note that since $\Gamma_{-}$commutes with $\widetilde{L}_{\psi}$, the form of the constraint $\Gamma_{-} \epsilon=0$ is identical for both $\epsilon$ and $\hat{\epsilon}$.

Note that in both of the latter two reductions, the constraint (84) reduces the number of supersymmetries by $1 / 2$. Furthermore in selecting out the $\epsilon_{+}$spinors, the reduced supersymmetries include those, i.e., $\epsilon_{+}^{-}$, that can be extended into the asymptotic regions of the full solution.

Having obtained the Killing spinors in the reduced solution we can determine the corresponding superalgebras by following the steps in section five and six. In both of the above cases, the Killing spinors have the form $\hat{\epsilon}_{+}$. The Killing vectors are then obtained by determining

$$
\overline{\hat{\epsilon}}_{+} \Gamma^{a} \hat{\epsilon}_{+} \tilde{\hat{e}}_{a}
$$

where the sum is now over all indices excluding the coordinate that one reduces on and the $\tilde{\hat{e}}_{a}$ are the dual vector fields in the reduced spacetime.

Let us first consider the reduction on $\partial_{w}$ to give $a d S_{2} \times S^{3} \times S^{3}$. Although the frame (35) without $e_{w}$ is a suitable frame for the reduced spacetime the dual vector fields (66) are not: instead we must now use $\tilde{\hat{e}}_{u}=Q \rho^{-2} \partial_{u}$. Taking this into account we find the Killing vectors as in (76) with the only difference being that we drop $\partial_{w}$ from $\ell_{+}^{L}$ in (77). This means that the superalgebra contains a factor $D(2 \mid 1, \alpha)$. Combining this with the bosonic symmetries that don't arise from Killing spinors, we conclude that the symmetry algebra is given by

$$
D(2 \mid 1, \alpha) \times S U(2) \times S U(2) .
$$

Next consider the reduction on $\partial_{\psi}$ to obtain $a d S_{3} \times S^{2} \times S^{3}$. We again obtain (76) but now with the Killing vectors $\xi_{L}$ replaced by Killing vectors obtained by setting $\psi=0$ and dropping $\partial_{\psi}$ terms in the expressions for $\xi_{L}$ in (68). In this case the symmetry algebra is $D(2 \mid 1, \alpha) \times S O(2,1) \times S U(2)$. 


\subsection{Adding Angular momentum}

Recall that the remnants of the angular momenta were eliminated by the coordinate transformation (28) in the near-horizon limit. After reduction on $w, \psi$ or $\psi^{\prime}$, such a transformation would not be allowed and so we should reconsider these reductions in the presence of the angular momentum. First, we must insert the angular momenta back in the metric (85). This is easily done as we simply undo part of the original coordinate transformation (28), reintroducing $\tilde{\psi}, \tilde{\psi}^{\prime}$

$$
\begin{aligned}
\psi & =\tilde{\psi}+\frac{2 \hat{J}}{R^{2}} \tilde{w} \\
\psi^{\prime} & =\tilde{\psi}^{\prime}+\frac{2 \hat{J}^{\prime}}{R^{\prime 2}} \tilde{w} \\
w & =\tilde{w}
\end{aligned}
$$

which in our angular forms yields

$$
\begin{aligned}
\sigma_{3} & =\tilde{\sigma}_{3}+\frac{2 \hat{J}}{R^{2}} d \tilde{w} \\
\sigma_{3}^{\prime} & =\tilde{\sigma}_{3}^{\prime}+\frac{2 \hat{J}^{\prime}}{R^{\prime 2}} d \tilde{w} .
\end{aligned}
$$

We distinguish $w$ and $\tilde{w}$ here because it will be necessary to distinguish the Killing vectors $\partial_{w}$ and $\partial_{\tilde{w}}$ later on. Now the metric (85) becomes

$$
d s_{11}^{2}=-\left(\frac{\rho^{2}}{Q} d u\right)^{2}+\left(Q d \tilde{w}-\frac{\rho^{2}}{Q} d u\right)^{2}+\frac{R^{2}}{4}\left(\tilde{\sigma}_{3}+\frac{2 \hat{J}}{R^{2}} d \tilde{w}\right)^{2}+\frac{R^{\prime 2}}{4}\left(\tilde{\sigma}_{3}^{\prime}+\frac{2 \hat{J}^{\prime}}{{R^{\prime 2}}^{2}} d \tilde{w}\right)^{2}+\ldots
$$

In this form, the metric is still adapted for a Kaluza-Klein reduction on $\tilde{\psi}$ (or $\tilde{\psi}^{\prime}$ ). A reduction on $k=\partial_{\tilde{\psi}}=\partial_{\psi}$ proceeds exactly as in the previous section. The only difference is that an extra gauge field $2 \hat{J} / R^{2} d \tilde{w}$ appears on the $a d S_{3}$ space. However, this is trivial since it is a constant gauge field.

The reduction on

$$
k=\partial_{\tilde{w}}=\partial_{w}+\frac{2 \hat{J}}{R^{2}} \partial_{\psi}+\frac{2 \hat{J}^{\prime}}{R^{\prime 2}} \partial_{\psi^{\prime}} .
$$

\footnotetext{
${ }^{3}$ Actually, at this point, we should note that the constraint equation (84) is coordinate invariant, as well as Lorentz invariant — see below.
} 
turns out to be more interestingf. For a Kaluza-Klein reduction on $\tilde{w}$, we must reorganize the metric (97) into the standard form. We do so by introducing new 1-forms

$$
\begin{aligned}
\hat{e}^{u} & =\frac{\rho^{2}}{\widetilde{Q}} d u-\frac{\hat{J}}{2 \widetilde{Q}} \tilde{\sigma}_{3}-\frac{\hat{J}^{\prime}}{2 \widetilde{Q}} \tilde{\sigma}_{3}^{\prime} \\
\hat{e}^{w} & =\widetilde{Q} d \tilde{w}-\frac{\rho^{2}}{\widetilde{Q}} d u+\frac{\hat{J}}{2 \widetilde{Q}} \tilde{\sigma}_{3}+\frac{\hat{J}^{\prime}}{2 \widetilde{Q}} \tilde{\sigma}_{3}^{\prime} \\
\hat{e}^{3} & =\frac{1}{2 s} \tilde{\sigma}_{3} \\
\hat{e}^{3^{\prime}} & =\frac{1}{2 c} \tilde{\sigma}_{3}^{\prime}
\end{aligned}
$$

with which the metric (97) may be written as

$$
d s_{11}^{2}=\left(\hat{e}^{w}\right)^{2}-\left(\hat{e}^{u}\right)^{2}+\left(\hat{e}^{3}\right)^{2}+\left(\hat{e}^{3^{\prime}}\right)^{2}+\ldots
$$

Here, $\widetilde{Q}^{2}=Q^{2}+(\hat{J} / R)^{2}+\left(\hat{J}^{\prime} / R^{\prime}\right)^{2}$. The reduced metric now comes from dropping $\left(\hat{e}^{w}\right)^{2}$ in (100) above. In this case, the remaining off-diagonal terms in $\left(\hat{e}^{u}\right)^{2}$ cannot be removed by a coordinate transformation, even locally. Hence the reduced geometry is not a simple product of factors. However, we will see that the structure of the isometry supergoup is identical, discounting changes in purely bosonic factors unrelated to Killing spinors, to that of the $J=J=0$ case, despite the fact that the metric has a direct product structure only in the $J=J^{\prime}=0$ limit.

To determine the surviving Killing spinors, we need to determine the constraint matrix $P$ in (84). The simplest approach, here, is to note that $P$ is coordinate invariant and Lorentz covariant. Hence the constraint will be the same as that calculated for (98) before any change of frames and coordinates. Furthermore, since $P$ is linear in $k$ and since we saw in the previous section that the constraints for reducing on $\partial_{w}, \partial_{\psi}$ and $\partial_{\psi^{\prime}}$ all coincided, precisely the same constraint arises here, namely, $\Gamma_{-} \epsilon=0$. Thus once again, the $\epsilon_{+}$Killing spinors correspond to supersymmetries in the reduced solution.

We must again be careful about the precise form of the Killing spinors in the reduced theory, as the frame (35) used in deriving (48) can not be used after the reduction. One

\footnotetext{
${ }^{4}$ The Killing vector $k$ will have closed orbits of radius $Q$ if the following identifications are made: $\left(w, \psi, \psi^{\prime}\right)=\left(w+2 \pi Q n_{1}, \psi+4 \pi n_{2}+\frac{4 \pi \hat{J} Q}{R^{2}} n_{1}, \psi^{\prime}+4 \pi n_{3}+\frac{4 \pi \hat{J}^{\prime} Q}{{R^{\prime}}^{2}} n_{1}\right)$, where $n_{i}$ are integers. As a result note that the global geometry of the unreduced space is no longer $a d S_{3} \times S^{3} \times S^{3} \times \mathbb{E}^{2}$.
} 
change which must be accounted for is the introduction of (99) for the Kaluza-Klein reduction. A second slightly more subtle change comes from the coordinate transformation (95) which introduces various $\tilde{w}$ dependences which we have not explicitly accounted for. First of all, in (48) and (49), one finds

$$
\begin{aligned}
\Omega(\psi, \theta, \phi) & =\exp \left(\frac{\hat{J} \tilde{w}}{R^{2}} \Gamma^{21}\right) \widetilde{\Omega}(\tilde{\psi}, \theta, \phi) \\
\Omega^{\prime}\left(\psi^{\prime}, \theta, \phi\right) & =\exp \left(\frac{\hat{J}^{\prime} \tilde{w}}{{R^{\prime}}^{2}} \Gamma^{2^{\prime} 1^{\prime}}\right) \widetilde{\Omega}^{\prime}\left(\tilde{\psi}^{\prime}, \theta^{\prime}, \phi^{\prime}\right) .
\end{aligned}
$$

Thus after the coordinate change the Killing spinors $\epsilon_{+}$depend on $\tilde{w}$. However in the same way, $\tilde{w}$ now also appears in the 1 -forms: $e^{1}, e^{2}, e^{1^{\prime}}$ and $e^{2^{\prime}}$. For example,

$$
e^{1}=\frac{R}{2}\left(-\sin \left(\tilde{\psi}+\frac{2 \hat{J} \tilde{w}}{R^{2}}\right) d \theta+\cos \left(\tilde{\psi}+\frac{2 \hat{J} \tilde{w}}{R^{2}}\right) \sin \theta d \phi\right) .
$$

Hence in the reduced theory, these 1-forms would be replaced by, e.g., , $\hat{e}^{1}, \hat{e}^{2}, \hat{e}^{1^{\prime}}$ and $\hat{e}^{2^{\prime}}$ defined using the $\sigma$-forms defined using $\tilde{\psi}$ - see (19). As in the $\psi$ reduction above, these two sets of 1 -forms are related by rotations acting in the 1-2 and $1^{\prime}-2^{\prime}$ subspaces. One finds

$$
\left(\begin{array}{c}
\hat{e}^{1} \\
\hat{e}^{2}
\end{array}\right)=L_{12}\left(\begin{array}{c}
e^{1} \\
e^{2}
\end{array}\right)
$$

with

$$
L_{12}=\left(\begin{array}{cc}
\cos \left(\frac{2 \hat{J} \tilde{w}}{R^{2}}\right) & \sin \left(\frac{2 \hat{J} \tilde{w}}{R^{2}}\right) \\
-\sin \left(\frac{2 \hat{J} \tilde{w}}{R^{2}}\right) & \cos \left(\frac{2 \hat{J} \tilde{w}}{R^{2}}\right)
\end{array}\right)=\exp \left[\left(\begin{array}{cc}
0 & 1 \\
-1 & 0
\end{array}\right) \frac{2 \hat{J}}{R^{2}} \tilde{w}\right]
$$

and similarly for $L_{1^{\prime} 2^{\prime}}$. The corresponding spinor rotations (90) are then

$$
\widetilde{L}_{12}=\exp \left(-\frac{\hat{J} \tilde{w}}{R^{2}} \Gamma^{21}\right) \quad \widetilde{L}_{1^{\prime} 2^{\prime}}=\exp \left(-\frac{\hat{J}^{\prime} \tilde{w}}{R^{\prime 2}} \Gamma^{21}\right)
$$

Hence this rotation, which removes the $\tilde{w}$ dependence in the angular frames, at the same time removes the $\tilde{w}$ dependence of the $\epsilon_{+}$spinors (and introduces it into the $\epsilon_{-}$). The net effect is that in $(48), \Omega \Omega^{\prime} \rightarrow \widetilde{\Omega} \widetilde{\Omega}^{\prime}$.

Now we also had to account for the change of frames (99). Since both (35) and (99) describe the same metric, they must be related by a Lorentz transformation. First one 
finds that in this four-dimensional subspace $\hat{e}^{a}=\left(L_{4}\right)^{a}{ }_{b} e^{b}$ with

$$
L_{4}=\left(\begin{array}{cccc}
z & z-z^{-1} & -x / z & -y / z \\
0 & z^{-1} & x / z & y / z \\
-x & -x & 1 & 0 \\
-y & -y & 0 & 1
\end{array}\right)
$$

where we have introduced the notation

$$
z=\frac{\widetilde{Q}}{Q}, \quad x=\frac{\hat{J} s}{Q}, \quad y=\frac{\hat{J}^{\prime} c}{Q} .
$$

The latter are not all independent, but rather from the definition of $\widetilde{Q}$, they satisfy the constraint: $z^{2}=1+x^{2}+y^{2}$.

To obtain the corresponding Lorentz transformation on the spinors as in (90), it is convenient to decompose $L_{4}$ as $L_{4}=B W$ where $B$ is a boost

$$
B_{b}^{a}=\left(\begin{array}{cccc}
\frac{1}{2}\left(z+z^{-1}\right) & \frac{1}{2}\left(z-z^{-1}\right) & 0 & 0 \\
\frac{1}{2}\left(z-z^{-1}\right) & \frac{1}{2}\left(z+z^{-1}\right) & 0 & 0 \\
0 & 0 & 1 & 0 \\
0 & 0 & 0 & 1
\end{array}\right)
$$

and $W$ is the remaining transformation

$$
W_{b}^{a}=\left(\begin{array}{cccc}
1+\frac{x^{2}+y^{2}}{2} & \frac{x^{2}+y^{2}}{2} & -x & -y \\
-\frac{x^{2}+y^{2}}{2} & 1-\frac{x^{2}+y^{2}}{2} & x & y \\
-x & -x & 1 & 0 \\
-y & -y & 0 & 1
\end{array}\right) .
$$

Now one finds $B=\exp \left(\omega_{B}\right)$ and $W=\exp \left(\omega_{W}\right)$ where

$$
\begin{aligned}
\left(\omega_{B}\right)_{a b}= & \left(\begin{array}{cccc}
0 & -\lambda & 0 & 0 \\
\lambda & 0 & 0 & 0 \\
0 & 0 & 0 & 0 \\
0 & 0 & 0 & 0
\end{array}\right) \\
\left(\omega_{W}\right)_{a b}= & \left(\begin{array}{cccc}
0 & 0 & x & y \\
0 & 0 & x & y \\
-x & -x & 0 & 0 \\
-y & -y & 0 & 0
\end{array}\right)
\end{aligned}
$$


with $\lambda=\log (z)$. Given these generators, we can write the corresponding spinor transformations: $\widetilde{L}=\widetilde{B} \widetilde{W}$ with

$$
\begin{aligned}
\widetilde{B} & =\exp \left(\frac{1}{2} \log z \Gamma^{w u}\right)=z^{1 / 2} \Lambda_{+}+z^{-1 / 2} \Lambda_{-} \\
\widetilde{W} & =\exp \left(\left(x \Gamma^{u 3}+y \Gamma^{u 3^{\prime}}\right) \Lambda_{+}\right) \\
& =1+\left(x \Gamma^{u 3}+y \Gamma^{u 3^{\prime}}\right) \Lambda_{+}
\end{aligned}
$$

where we have defined the projection operators

$$
\Lambda_{ \pm}=\frac{1}{2}\left(1 \pm \Gamma^{w u}\right)
$$

So given the original Killing spinor solutions (47) and (48), they are transformed to the new frame by

$$
\hat{\epsilon}=\widetilde{L}_{12} \widetilde{L}_{1^{\prime} 2^{\prime}} \widetilde{L}_{4} \epsilon=\widetilde{L}_{12} \widetilde{L}_{1^{\prime} 2^{\prime}} \widetilde{B} \widetilde{W} \epsilon
$$

Given that all of these transformation matrices commute with $\Gamma_{-}$, the constraint $\Gamma_{-} \epsilon=0$ takes precisely the same form on the $\hat{\epsilon}$ spinors. Hence the supersymmetries of the solution in the reduced theory are given by $\hat{\epsilon}_{+}$. Explicitly (113) yields

$$
\hat{\epsilon}_{+}=\left(\frac{1}{\rho}\left[z^{1 / 2}+z^{-1 / 2}\left(x \Gamma^{u 3}+y \Gamma^{u 3^{\prime}}\right)\right]-\frac{2 u \rho}{\sqrt{z} \gamma Q} \Gamma^{\rho u}\right) \widetilde{\Omega} \widetilde{\Omega}^{\prime} \chi_{+}^{+}+\frac{\rho}{\sqrt{z}} \widetilde{\Omega} \widetilde{\Omega}^{\prime} \chi_{+}^{-}
$$

Note that the Killing spinor $\hat{\epsilon}_{+}^{-}$, which should correspond to the supersymmetry which extends to the full rotating solution in the reduced theory, still has essentially the same simple form as with $J=J^{\prime}=0$.

Having established the explicit form of the Killing spinors we deduce that the full isometry superalgebra in the reduced spacetime is now $D(2 \mid 1, \alpha) \times U(1) \times U(1)$. Recalling eq. (94) we see that the effect of the rotation in each of the two 4-planes is to break the extra bosonic $S U(2)$ rotational symmetries of these 4-planes to $S O(2) \cong U(1)$, without affecting the supersymmetry.

\section{Discussion}

We have found a new family of $1 / 8$ supersymmetric rotating M-brane solutions, with two independent rotation parameters. Many previous supersymmetric rotating brane 
solutions can be found from the $\alpha \rightarrow 0$ limit of this new solution by a combination of dualities and compactifications. The near-horizon limit of the new rotating brane solution is the $1 / 2$ supersymmetric $a d S_{3} \times S^{3} \times S^{3} \times \mathbb{E}^{2}$ vacuum, irrespective of the rotation parameters (within the limits for which there exists a non-singular event horizon). There is thus a fourfold increase of supersymmetry near the horizon, although this is invariably reduced to a two-fold increase on $S^{1}$ compactification by the identifications required to perform the reduction. Non-trivial $S^{1}$ compactifications lead to a replacement of $a d S_{3}$ by $a d S_{2}$ or $S^{3}$ by $S^{2}$ in the near-horizon limit. The possible near-horizon geometries obtainable this way were classified in [9] for non-rotating intersecting branes; we now see that the same results apply in the rotating case, at least locally.

Finally we note that the $(1 \mid M W, M 2, M 5, M 5)$ configuration of M-theory has a IIB dual as $(1 \mid I I W, D 1, D 5, D 5)$ so that the entropy associated with the event horizon is expected to correspond to a counting of D-brane microstates along the lines of [20]. It would be of interest to see how the rotation affects these calculations. We leave this to future investigation.

Acknowledgements: JPG would like to thank the ITP for hospitality during the workshop "Dualities in String Theory" where this work began, and the EPSRC for partial support. RCM was supported in part by NSERC of Canada and Fonds FCAR du Québec. Research at the ITP was supported by NSF Grant PHY94-07194.

\section{References}

[1] G.W. Gibbons and P.K. Townsend, Phys. Rev. Lett. 71 (1993) 3754 hepth/9307049].

[2] G. Papadopoulos and P.K. Townsend, Phys. Lett. B380 (1996) 273 hepth/9603087];

A.A. Tseytlin, Nucl. Phys. B475 (1996) 149 hep-th/9604035;

J.P. Gauntlett, D. Kastor and J. Traschen, Nucl. Phys. B478 (1996) 544 hepth/9604179]. 
[3] M.J. Duff, G.W. Gibbons and P.K. Townsend, Phys. Lett. 332 B (1994) 321 hepth/9405124].

[4] H.J. Boonstra, B. Peeters and K. Skenderis, Phys. Lett. 411B (1997) 59, hepth/9706192.

[5] J.P. Gauntlett, G.W. Gibbons, G. Papadopoulos and P.K. Townsend, Nucl. Phys. B500 (1997) 133 hep-th/9702202.

[6] A. A. Tseytlin, Class.Quant.Grav. 14 (1997) 2085.

[7] I. Antoniadis, C. Bachas and A. Sagnotti, Phys. Lett. 235B (1990) 255;

A. Chamseddine and M.S. Volkov, Phys. Rev. D57 (1998) 6242, hep-th/9711181.

[8] P. Cowdall and P.K. Townsend, Phys. Lett. B429 (1998) 281, hep-th/9801165.

[9] H.J. Boonstra, B. Peeters and K. Skenderis, Brane intersections, anti-de Sitter Spacetimes and dual superconformal theories, hep-th/9803231.

[10] A. Sevrin, W. Troost and A. van Proeyen, Phys. Lett. 208B (1988) 447.

[11] G.T. Horowitz and D. Marolf, Phys. Rev. D55 (1997) 835 hep-th/9605224.

[12] J.C. Breckenbridge, R.C. Myers, A.W. Peet and C. Vafa, Phys. Lett. B391 (1997) 93 hep-th/9602065.

[13] J.C. Breckenridge, D.A. Lowe, R.C. Myers, A.W. Peet, A. Strominger and C. Vafa, Macroscopic and microscopic entropy of near-extremal spinning black holes, hepth/9603078.

[14] M. Cvetič and F. Larsen, Near-horizon geometry of rotating black holes in five dimensions, hep-th/9805097; Microstates of four-dimensional rotating black holes from near horizon geometry, hep-th/9805146.

[15] M. Cvetič and D. Youm, Nucl. Phys. B499 (1997) 253, hep-th/9612229.

[16] E. Cremmer, B. Julia and J. Scherk, Phys. Lett. 76B (1978) 409. 
[17] A. A. Tseytlin, Mod.Phys.Lett. A11 (1996) 689, hep-th/9601177

[18] J. Maldacena, The Large N Limit of Superconformal Field Theories and Supergravity, hep-th/9711200.

[19] B. de Witt, Supermanifolds, Cambridge University Press, 1984.

[20] A. Strominger and C. Vafa, Phys.Lett. B379 (1996) 99, hep-th/9601029

C.G. Callan and J.M. Maldacena, Nucl.Phys. B472 (1996) 591, hep-th/9602043. 